\title{
Colonization pattern of Gram positive organisms causing neonatal sepsis in pregnant women
}

\author{
Hamima Hasnat ${ }^{1}$, Sadia Afroz ${ }^{2}$, Faijul Islam ${ }^{3}$, Mohammad Monir Hossain ${ }^{4}$, Shikha Paul ${ }^{2}$ \\ ${ }^{1}$ Department of Microbiology, Gonoshasthaya Samaj Vittik Medical College, Savar, Dhaka, Bangladesh, ${ }^{2}$ Department of \\ Microbiology, Sir Salimullah Medical College, Dhaka, Bangladesh, ${ }^{3}$ National Institute of Mental Health, Dhaka, Bangladesh, \\ ${ }^{4}$ Department of Anatomy, Eastern Medical College, Cumilla, Bangladesh.
}

\begin{abstract}
Background: Neonatal sepsis is one of the major causes of mortality and morbidity in neonates. Early onset neonatal sepsis (EONS) is commonly caused by mother's vaginal and rectal organisms before or during the delivery process.
\end{abstract}

Objective: The aim of this study was to determine the colonization pattern of common gram positive organisms responsible for neonatal sepsis, in women of 35-37 weeks of pregnancy.

Methodology: A cross sectional analytical study was conducted from July 2018 to June 2019 at the department of Microbiology of Sir Salimullah Medical College, Dhaka. Total 107 of each vaginal and vaginorectal samples was collected from pregnant women at 35-37 weeks of pregnancy attending at the out-patient department of the Obstetrics and Gynaecology unit of Sir Salimullah Medical College \& Mitford Hospital, Dhaka. Vaginal and vaginorectal samples were tested by standard culture technique using Todd- Hewitt broth, blood agar, High chrome UTI agar media. Data was collected by a questionnaire and results were analyzed by statistical package for the social sciences (SPSS) program.

Results: In this study, among 107 vaginal swab samples Staphylococcus aureus were the predominant isolate (51.40\%), followed by Enterococci (20.56\%), Coagulase negative Staphylococcus (14.95\%) and Group B Sreptococcus (5.61\%). Similarly among 107 vaginorectal swab samples commonest isolates were Staphylococcus aureus (56.1\%), followed by Enterococci (52.34\%), Coagulase negative Staphylococcus (11.21\%) and Group B Streptococcus (6.54\%).

Conclusion: The organisms from the vaginal and vaginorectal swabs were essentially the same. Staphylococcus aureus and Enterococci were the most abundant organisms that can cause neonatal sepsis, isolated from the vaginal and vaginorectal swab samples.

\section{Introduction}

Neonatal sepsis is one of the important cause of mortality and morbidity of newborns and is more common in developing countries compared with developed countries ${ }^{1}$. In developing countries the incidence of sepsis is higher (1.8-18/1000) than the incidence in developed countries $(1-5 / 1000)^{2}$.

In Bangladesh neonatal mortality rate (NMR) is $27 / 1000$ live birth and among this neonatal sepsis contributes to

\footnotetext{
Correspondence:

Dr. Hamima Hasnat

Assistant Professor, Department of Microbiology

Gonoshasthaya Samaj Vittik Medical College, Savar, Dhaka

Mobile: 01812-094060

Email: hamimahasnat@yahoo.com
}

about $36 \%$ of total neonatal death. Neonatal sepsis has been classified as either early onset sepsis (EOS), presenting at 0-7 days of age, or late onset sepsis (LOS), occurring at 7-28 days of age ${ }^{1,3}$. EOS is due to vertical transmission during labor or birth. Maternal rectovaginal organisms play an important role in the incidence of early neonatal sepsis. LOS is due to vertical, horizontal or nosocomial infection ${ }^{4,5}$.

Several bacterial species are known to colonize both the gastrointestinal and the reproductive tract, and the rectum has been suggested to play an important role as a source or reservoir for organisms that colonize the vagina ${ }^{6}$. Among gram positive bacteria Group B Streptococcus (Strepto- 
coccus agalactiae, GBS), Staphylococcus aureus, coagulase -negative Staphylococci (CONS) and Enterococcus spp., are the most common rectovaginal colonizer and are known to be associated with neonatal sepsis ${ }^{7,8}$. According to data from the American Neonatology Network, gram-positive microorganisms are the etiological agents in $62 \%$ of early neonatal sepsis cases and in $79 \%$ of late neonatal sepsis ${ }^{8,9}$.

Group B Streptococcus (GBS) has been the most frequent etiological agent of neonatal sepsis in developed countries, being responsible for high morbidity and mortality ${ }^{10}$. Upto $30 \%$ adults, are commonly colonized with GBS in the gastrointestinal and genital tract but remain asymptomatic $^{11}$. The gastrointestinal tract serves as the primary reservoir for GBS and is the likely source of vaginal or rectal colonization ${ }^{12}$.

Women of childbearing age carry GBS at variable frequencies of 4.6\%-31.3\% in both developing and developed countries ${ }^{13}$. Pregnant women who are carriers of GBS have $40 \%-73 \%$ potential capacity for vertical transmission of the microorganism and $1 \%-2 \%$ of their newborns develop invasive GBS infection, with significant mortality (5\%-20\%) and morbidity, especially among premature neonates ${ }^{14,15}$.

The rates of colonization of $S$. aureus in the women of reproductive age group range from 5\% to $26 \%{ }^{16}$. Maternal colonization increases the risk of newborn staphylococcal colonization, through vertical transmission and breastfeeding ${ }^{17}$. S. aureus is a rare cause of early-onset sepsis (EOS) $(<1 \%)$ and responsible for $8 \%$ to $15 \%$ of $\operatorname{LOS}^{18}$. Overall, $S$. aureus mortality ranges from $5 \%$ to $18 \%$, but it is as high as $25 \%$ in very low birth weight infants (VLBW; <1500 g BW) ${ }^{19}$.

CoNS is a common vaginal colonizer. It is most important bacterial cause of sepsis and meningitis in newborns ${ }^{20}$. $2 \%$ to $22.5 \%$ of EOS are caused by CoNS and $50 \%$ of LOS are caused by CoNS ${ }^{18,21}$. Infants born prematurely are at increased risk of CoNS sepsis ${ }^{22}$. Neonatal sepsis and meningitis with coagulase-negative Staphylococci are associated with significant morbidity and have a mortality rate as high as $10 \%{ }^{23}$.

Enterococci are among the first bacteria that colonize the neonatal gastrointestinal tract either through oral ingestion of breast milk or from the vaginal and gastrointestinal flora of the mother during the delivery. Enterococcus spp., are the most important cause of nosocomial infections and are the second most common cause of urinary tract infections (UTI) in hospitals. It also causes endocarditis, postpartum endometritis, abortion and neonatal sepsis ${ }^{23,24}$. It was estimated that $10 \%$ cases of neonatal bacteraemia and septicaemia are caused by Enterococci. This infection can be acquired from the the vaginal flora of the mother or as cross infection from the hospital ${ }^{23}$.

The aim of this study was to determine the common rectovaginal colonization of gram positive organisms that can cause neonatal sepsis, in women of 35-37 weeks of pregnancy, in order to decide the most effective empirical antibiotic therapy for neonatal sepsis.

\section{Materials and Methods}

Department of Microbiology, Sir Salimullah Medical College, Dhaka. Study population included pregnant women of 35-37 weeks attending at the out-patient department of the Obstetrics and Gynaecology unit of Sir Salimullah Medical College \& Mitford Hospital, Dhaka. Those excluded from the study were: Pregnant woman of $<35$ weeks and $>37$ weeks of gestation, rupture of membrane at the time of sample collection, pregnant women with per vaginal bleeding at the time of sample collection, patient under antimicrobial therapy or history of intake of antibiotics during the past 2 weeks, preexisting medical disorders complicating pregnancy, women with urinary tract infection or vaginal infection in the current pregnancy and patients who did not give consent for this study. In this study, 214 samples (one sample from the vagina and one sample from the vaginorectal) were taken from 107 women at 35-37 weeks of pregnancy. A questionnaire and a check list were the tools of data collection. Informed written consent was taken from each patient or attendant. Before collecting specimen, each patient was interviewed and relevant information was recorded systematically in a pre-designed standard data sheet and data was analyzed by appropriate method. The study was approved by protocol approval committee \& Ethical review committee of Sir Salimullah Medical College, Dhaka. This cross sectional analytical study was conducted from July 2018 to June 2019.

\section{Collection of samples}

Two samples were collected from each patient with strict aseptic precaution. One vaginal swab was obtained from the lower third of the vagina by one sterile swab stick. 
Subsequently, the swab was carefully withdrawn to prevent contamination with microflora from the vulva and introitus. Then one vaginorectal swabs was obtained from the lower third of the vagina by one sterile swab stick. Subsequently, the swab was carefully withdrawn to prevent contamination with microflora from the vulva and introitus and the swab was inserted 1.5 to $2 \mathrm{~cm}$ beyond the anal sphincter and gently rotated to touch the anal crypts. Vaginal and vaginorectal swab were immediately inoculated into $5 \mathrm{ml}$ of Todd-Hewitt broth with $10 \mu \mathrm{g}$ colistin $/ \mathrm{ml}$ at the bed side. Then all swabs were transported to the laboratory within 4 hours ${ }^{25}$.

\section{Culture}

The swabs were inoculated into Todd-Hewitt broth media. The Inoculated Todd-Hewitt broth was incubated for $18-24$ hours at $37^{\circ} \mathrm{C}^{26}$. Then subculture was done into Blood Agar media, MacConkey agar media, HiChrome UTI agar and Chocolate agar media. Blood Agar media, and Chocolate agar media was incubated for 18-24 hours at $37^{\circ} \mathrm{C}$ with $5 \% \mathrm{CO}_{2}$ (by using a candle jar, $5 \% \mathrm{CO}_{2}$ was generated). MacConkey agar media and HiChrome UTI agar was incubated for $18-24$ hours at $37^{\circ} \mathrm{C}$. At the end of incubation the culture plates were examined and appropriate colonies were subcultured for pure isolation. If no growth was found, the plates were incubated for an additional 24 hours and re-examined for growth of the organism. If no growth was found on the second examination, the plates were declared as negative. Identification of organisms was performed based on colony morphology, Gram stain, catalase test, CAMP test and bacitracin sensitivity test ${ }^{27}$.

\section{Statistical analysis}

All necessary information and clinical data were systematically recorded in a pre-designed data collection sheet. Data analysis was done by SPSS version 17.

\section{Results}

Out of 107 vaginal swab samples $93(86.92 \%)$ yielded bacterial growth and no growth occurred in 14 (13.08\%) cases by culture and among 107 vaginorectal swab samples, all (100\%) yielded bacterial growth by culture. (Fig: I).

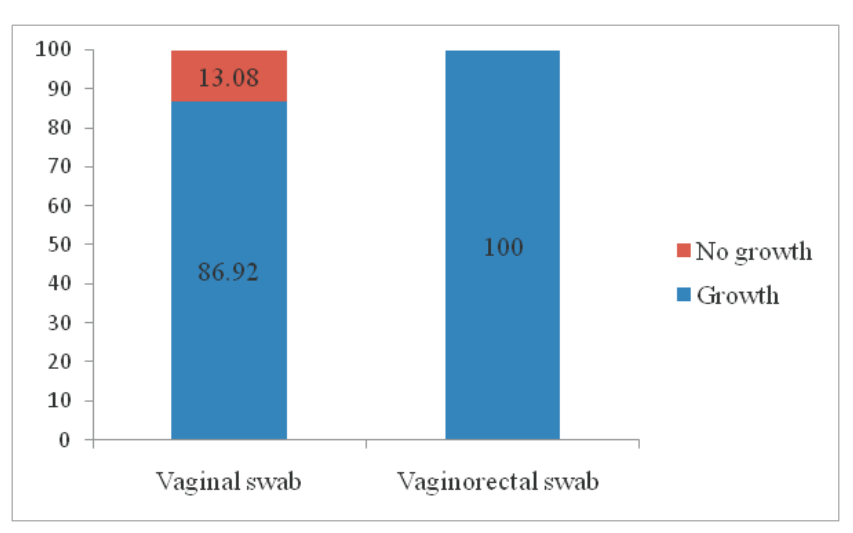

Fig I: Percentage of bacterial growth from vaginal and vaginorectal swab among study population by culture.

Among 107 vaginal swab samples, Staphylococcus aureus was the most commonly isolated organism 55 (51.40\%), followed by Enterococci 22 (20.56\%) and Coagulase negative Staphylococcus 16 (14.95\%). While Group B Sreptococcus were isolated from 6 (5.61\%) vaginal swab samples (Table I).

Table I: Frequency of isolated microorganisms from vaginal swab by culture $(n=107)$

\begin{tabular}{lcc}
\hline Organisms & Number & Percentage (\%) \\
\hline Staphylococcus aureus & 60 & 56.1 \\
Enterococci & 56 & 52.34 \\
Coagulase negative & 12 & 11.21 \\
Staphylococcus & & \\
Group B Streptococcus & 7 & 6.54 \\
\hline
\end{tabular}

Almost similar pattern was found in vaginorectal swab samples. Among 107 vaginorectal swab samples, Staphylococcus aureus was the commonest organism 60 (56.1\%), followed by Enterococci 56 (52.34\%) and Coagulase negative Staphylococcus 12 (11.21\%). Group B Streptococcus were isolated from 7(6.54\%) samples (Table II).

Table II: Frequency of isolated microorganisms from vaginorectal swab by culture $(n=107)$

\begin{tabular}{lcc}
\hline Organisms & Number & Percentage (\%) \\
\hline Staphylococcus aureus & 55 & 51.40 \\
Enterococci & 22 & 20.56 \\
Coagulase negative & 16 & 14.95 \\
Staphylococcus & & \\
Group B Streptococcus & 6 & 5.61 \\
\hline
\end{tabular}




\section{Discussion}

Neonates are more vulnerable to infection in the first week of life. It is estimated that infection causes $42 \%$ of deaths in the first week of life ${ }^{28}$. Early onset neonatal sepsis is generally caused by microorganisms obtained from the mother before or during the delivery ${ }^{29}$. Rectovaginal colonization of pregnant women is suspected to be a risk factor for neonatal sepsis ${ }^{30}$.

The microorganisms that cause early-onset sepsis may vary depending on time and region across the world. Some studies found that gram positive bacteria were the commonest cause of neonatal sepsis ${ }^{31}$ while one study showed that the frequency of isolation of both gram positive and gram negatives was equal ${ }^{32}$.

In the present study, 107 vaginal and 107 vaginorectal swab were collected from pregnant women at 35-37 weeks and different microorganisms were isolated and identified from vaginal and vaginorectal swab. All $(100 \%)$ vaginorectal swab yielded bacterial growth, while in case of vaginal swab 93 (86.92\%) showed bacterial growth and $14(13.08 \%)$ were culture negative. Vaginorectal swab was found to be better sampling technique for detection of colonization in pregnant women. This observation is consistent with other studies, which reported that vaginorectal sample is more accurate than vaginal sample ${ }^{33,34}$.

In this study, isolated organisms from vaginal swab that can cause neonatal sepsis, were Staphylococcus aureus (51.40\%), Enterococcus (20.56\%), Coagulase negative Staphylococcus (14.95\%), Group B Streptococcus (5.61\%). These findings correlate with the findings of Ekanem et $\mathrm{al}^{35}$. where the commonly found organisms were, Coagulase negative staphylococcus (58.6\%), Staphylococcus aureus (53.1\%), Enterococcus (40.0\%), Group B Streptococcus (12.2\%). Studies carried out by Tarana and Shamsuzzaman ${ }^{36}$, reported that Staphylococcus aureus (19.64\%), Group B Streptococcus (12.50\%), Coagulase negative Staphylococcus $(3.57 \%)$ were the most frequent organisms isolated from vaginal swab. Shirazi et $\mathrm{al}^{37}$. in their study found a similar pattern of organisms. So it was evident that, although the types of colonizing organisms were similar but the isolation rate of organisms were different in various studies, this may be due to, different laboratory methods used in those studies. Moreover the composition of the vaginal flora is not static but changes over time in response to several endogenous and exogenous influences ${ }^{38}$.
In this study, isolated organisms from vaginorectal swab were Staphylococcus aureus (56.1\%), Enterococcus (52.34\%), Coagulase negative Staphylococcus (11.21\%), Group B Streptococcus (6.54\%). These findings correlate with the findings of Hassanzadeh et $\mathrm{al}^{39}$. where the commonly found organisms were, Coagulase negative Staphylococcus (26.4\%), Staphylococcus aureus (7\%), Enterococcus (25.1\%), Group B Streptococcus (13.8\%). Another study carried out by Javanian et $\mathrm{al}^{40}$. reported that Coagulase negative Staphylococcus (55.8\%), Group B Streptococcus $(11.7 \%)$ were the most frequent organisms isolated from vaginorectal swab. In this study there is no significant difference between the isolated gram positive organisms from vaginal and vaginorectal swabs. These organisms colonize in the gastrointestinal tract and due to close proximity of anus these organisms may migrate to genital tract and consequently were isolated from vaginal and vaginorectal swabs ${ }^{41,42,43}$.

According to the present study, Staphylococcus aureus and Enterococcus spp. were the most common rectovaginal colonizer among the women of 35-37 weeks of pregnancy that can be the potential source of neonatal sepsis.

\section{Conclusion}

The findings of the present study revealed that Staphylococcus aureus and Enterococcus spp. were the most common isolated organisms among the women of 35-37 weeks of pregnancy. Early-onset infections that are caused by organisms prevalent in the maternal genital tract can be prevented through screening program for maternal vaginorectal colonization. Available programs include detection of GBS colonization in the last gestational weeks. These programs allow the reduction of GBS infections in newborns but are not directed to the detection of other pathogens involved in EONS. So the extension of the screening program to other pathogens could identify them and helps to implement the administration of specific antibiotic prophylaxis.

\section{References}

1. Vergnano S, Sharland M, Kazembe P, Wansambo CM, Heath PT. Neonatal sepsis: an international perspective. ADC fetal \& Neonatal edition. 2005 May 1;90(3):F220-F224.

2. Child Health Research. Project special report: Reducing perinatal and neonatal mortality, report of a meeting. Baltimore, Maryland. 1999; 6-12. 
3. Kaftan H, Kinney JS. Early onset neonatal bacterial infections. Seminars in perinatology. 1998 Feb 1; 22(1):15-24.

4. Raha BK, Baki MA, Begum T, Nahar N, Jahan N, Begum M. Clinical, bacteriological profile \& outcome of neonatal sepsis in a tertiary care hospital. Medicine today. 2014 Dec 25;26(1):18-21.

5. Javanian M, Rad ZA, Mojaveri MH, Shiadeh AG, Ebrahimpour S. Maternal recto vaginal colonization in term and preterm deliveries. Electronic physician. 2017 Oct;9(10):5434.

6. Antonio MA, Rabe LK, Hillier SL. Colonization of the rectum by Lactobacillus species and decreased risk of bacterial vaginosis. The Journal of infectious diseases. 2005 Aug 1;192(3):394-8.

7. Ghasemi E, Mansouri S, Shahabinejad N. Vaginal colonization and susceptibility to antibiotics of enterococci during late pregnancy in Kerman City, Iran. Archives of clinical infectious diseases. 2016 Oct $1 ; 11(4)$.

8. Stoll BJ, Hansen NI, Sánchez PJ, Faix RG, Poindexter BB, Van Meurs KP, et al. Early onset neonatal sepsis: the burden of group B Streptococcal and E. coli disease continues. Pediatrics. 2011 May 1;127(5):817-26.

9. Greenberg RG, Kandefer S, Do BT, Smith PB, Stoll $\mathrm{BJ}$, Bell EF,et al. Late-onset sepsis in extremely premature infants: 2000-2011. The pediatric infectious disease journal. 2017 Aug;36(8):774..

10. Freedman RM, Ingram DL, Gross I, Ehrenkranz RA, Warshaw JB, Baltimore RS. A half century of neonatal sepsis at Yale: 1928 to 1978. The American journal of diseases of children. 1981 Feb 1;135(2):140-4.

11. Melin P. Neonatal group B streptococcal disease: from pathogenesis to preventive strategies. Clinical microbiology and infection. 2011 Sep 1;17(9):1294-303.

12. Verani JR, McGee L, Schrag SJ. Prevention of perinatal group B streptococcal disease. Morbidity and Mortality Weekly Report (MMWR), Revised Guidelines from CDC, Recommendations and Reports. 2010 Jun 18;59(RR10):1-32.

13. Bidgani S, Navidifar T, Najafian M, Amin M. Comparison of group B streptococci colonization in vaginal and rectal specimens by culture method and polymerase chain reaction technique. Journal of the Chinese medical association. 2016 Mar 1;79(3):141-5.

14. Darabi R, Tadi S, Mohit M, Sadeghi E, Hatamizadeh $\mathrm{G}$, Kardeh B, et al.The prevalence and risk factors of group B streptococcus colonization in Iranian pregnant women. Electronic physician. 2017 May;9(5):4399.

15. Sridhar Santhanam RJ, Sahni RD, Thomas N, Beck MM. Prevalence of group B Streptococcal colonization among pregnant women and neonates in a tertiary hospital in India. Journal of the Turkish German gynecological association. 2017 Dec;18(4):181.

16. Parsonnet J, Hansmann MA, Delaney ML, Modern PA, DuBois AM, Wieland-Alter W, et al. Prevalence of toxic shock syndrome toxin 1-producing Staphylococcus aureus and the presence of antibodies to this superantigen in menstruating women. Journal of clinical microbiology. 2005 Sep 1;43(9):4628-34.

17. Jimenez-Truque N, Tedeschi S, Saye EJ, McKenna BD, Langdon W, Wright JP, et al. Relationship between maternal and neonatal Staphylococcus aureus colonization. Pediatrics. 2012 May 1;129(5): e1252-9.

18. Stoll BJ, Hansen N, Fanaroff AA, Wright LL, Carlo WA, Ehrenkranz RA, et al. Late-onset sepsis in very low birth weight neonates: the experience of the NICHD Neonatal Research Network. Pediatrics. 2002 Aug 1;110(2):285-91

19. Blanchard AC, Quach C, Autmizguine J. Staphylococcal infections in infants: updates and current challenges. Clinics in perinatology. 2014 Nov 28;42(1):119-32.

20. Ranjit E, Raghubanshi BR, Maskey S, Parajuli P. Prevalence of bacterial vaginosis and its association with risk factors among nonpregnant women: A hospital based study. International journal of microbiology. 2018 Mar 5;2018.

21. Sridhar Santhanam RJ, Sahni RD, Thomas N, Beck MM. Prevalence of group B Streptococcal colonization among pregnant women and neonates in a tertiary hospital in India. Journal of the Turkish German gynecological association. 2017 Dec;18(4):181.

22. Healy CM, Baker CJ, Palazzi DL, Campbell JR, Edwards MS. Distinguishing true coagulase-negative Staphylococcus infections from contaminants in the neonatal intensive care unit. Journal of perinatology. 2013 Jan;33(1):52-8. 
23. Shantala GB, Nagarathnamma T, Pooja DR, Harsha TR, Karthik R. Neonatal septicaemia caused by vancomycin resistant enterococcus faecium-a case report. Journal of clinical and diagnostic research for doctors. 2014 Nov;8(11):DD03.

24. da Silva NS, Muniz VD, Estofolete CF, Furtado GH, Rubio FG. Identification of temporal clusters and risk factors of bacteremia by nosocomial vancomycinresistant enterococci. American journal of infection control. 2014 Apr 1;42(4):389-92.

25. Sadaka SM, Aly HA, Meheissen MA, Orief YI, Arafa BM. Group B streptococcal carriage, antimicrobial susceptibility, and virulence related genes among pregnant women in Alexandria, Egypt. Alexandria journal of medicine. 2018 Apr 19;54(1):69-76.

26. Baker CJ, Clark DJ, Barrett FF. Selective broth medium for isolation of group B streptococci. Applied microbiology. 1973 Dec;26(6):884-5.

27. Cheesbrough M. Culturing Blood. In: District laboratory practice in tropical countries. Part 2. 2nd ed. New Delhi: Cambridge university press; 2006. p. 124-129.

28. Thaver D, Zaidi AK. Burden of neonatal infections in developing countries: a review of evidence from community-based studies. The Pediatric infectious disease journal. 2009 Jan 1;28(1):S3-9.

29. Kerur BM, Bhat BV, Harish BN, Habeebullah S, Kumar CU. Maternal genital bacteria and surface colonization in early neonatal sepsis. The Indian journal of pediatrics. 2006 Jan;73(1):29-32.

30. Seale AC, Blencowe H, Manu AA, Nair H, Bahl R, Qazi SA, et al. Estimates of possible severe bacterial infection in neonates in sub-Saharan Africa, south Asia, and Latin America for 2012: a systematic review and meta-analysis. The Lancet infectious diseases. 2014 Aug 1;14(8):731-41.

31. Anwer SK, Mustafa S, Pariyani S, Ashraf S, Taufiq KM. Neonatal sepsis: an etiological study. Journalpakistan medical association. 2000 Mar 1;50(3):91-3.

32. Dawodu A, Al Umran K, Twum-Danso K. A case control study of neonatal sepsis: experience from Saudi Arabia. Journal of tropical pediatrics. 1997 Apr $1 ; 43(2): 84-8$.

33. El Aila NA, Tency I, Claeys G, Saerens B, Cools P, Verstraelen $\mathrm{H}$, et al. Comparison of different sampling techniques and of different culture methods for detection of group B streptococcus carriage in pregnant women. BMC infectious diseases. 2010 Dec;10(1):1-8.

34. Quinlan JD, Hill DA, Maxwell BD, Boone S, Hoover F, Lense JJ. The necessity of both anorectal and vaginal cultures for group B streptococcus screening during pregnancy. Journal of family practice. 2000 May $1 ; 49(5): 447$.

35. Ekanem E, Efiok E, Udoh A, Inyang-Out A. Study of the bacterial flora of the vagina and cervix in women of childbearing age in rural community of Niger Delta Region, Nigeria. Obstetrics and gynaecology 2012;2(1):1-4.

36. Tarana MN, Shamsuzzaman SM. Detection of Group B Streptococcus in Vaginal Swab of Pregnant Women by Culture and PCR and Their Antibiotic Susceptibility Pattern in a Tertiary Care Hospital of Bangladesh. Mymensingh medical journal. 2018 Jul 1;27(3): 567-72.

37. Shirazi M, Abbariki E, Hafizi A, Shahbazi F, Bandari M, Dastgerdy E. The prevalence of group B treptococcus colonization in Iranian pregnant women and its subsequent outcome. International journal of fertility \& sterility. 2014 Jan;7(4):267.

38. Bartlett JG, Moon NE, Goldstein PR, Goren B, Onderdonk AB, Polk BF. Cervical and vaginal bacterial flora: ecologic niches in the female lower genital tract. American journal of obstetrics and gynecology. 1978 Mar 15;130(6):658-61.

39. Hassanzadeh P, Motamedifar M, Gharaghani MN. Carriage rate of group B streptococci in pregnant women in three teaching hospitals in Shiraz, Iran. Medical principles and practice 2011;20(3):277-82.

40. Javanian M, Rad ZA, Mojaveri MH, Shiadeh AG, Ebrahimpour S. Maternal recto vaginal colonization in term and preterm deliveries. The electronic physician 2017 Oct;9(10):5434.

41. Febriani AD, Handriyati A, Alasiry E, Daud D. The correlation between the mothers vaginal bacterial colonization and incidence of early onset neonatal sepsis. Current pediatric research 2017; 21(1): 105-111. 
42. Macfarlane S, Macfarlane GT. Composition and metabolic activities of bacterial biofilms colonizing food residues in the human gut. Applied and environmental microbiology 2006 Sep 1;72(9):6204-11.

43. Akinkunmi EO, Adeyemi OI, Igbeneghu OA, Olaniyan EO, Omonisi AE, Lamikanra A. The pathogenicity of Staphylococcus epidermidis on the intestinal organs of rats and mice: an experimental investigation. BMC gastroenterology. 2014 Dec 1;14(1):126. 